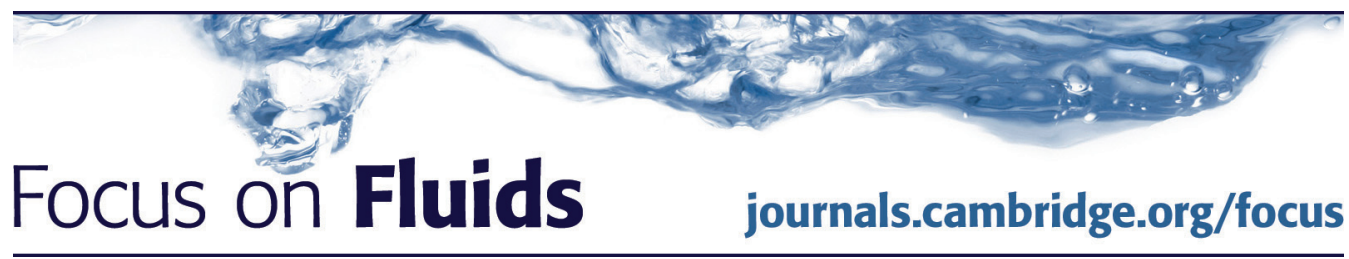

\title{
Mixed thermal conditions in convection: how do continents affect the mantle's circulation?
}

\section{R. Ostilla-Mónico $†$}

John A. Paulson School of Engineering and Applied Sciences and Kavli Institute for Bionano Science and Technology, Harvard University, Cambridge, MA 02138, USA

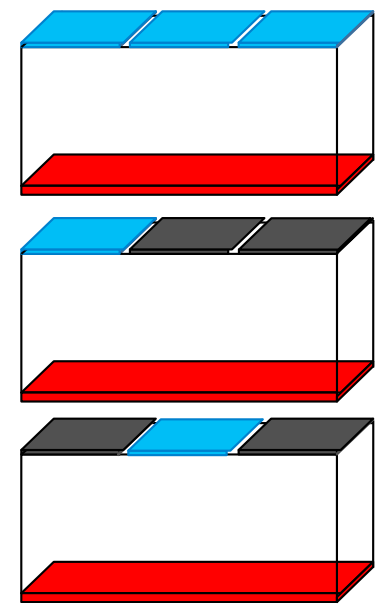

Natural convection is omnipresent on Earth. A basic and well-studied model for it is Rayleigh-Bénard convection, the fluid flow in a layer heated from below and cooled from above. Most explorations of Rayleigh-Bénard convection focus on spatially uniform, perfectly conducting thermal boundary conditions, but many important geophysical phenomena are characterized by boundary conditions which are a mixture of conducting and adiabatic materials. For example, the differences in thermal conductivity between continental and oceanic lithospheres are believed to play an important role in plate tectonics. To study this, Wang et al. (J. Fluid Mech., vol. 817, 2017, R1), measure the effect of mixed adiabatic-conducting boundary conditions on turbulent Rayleigh-Bénard convection, finding experimental proof that even if the total heat transfer is primarily affected by the adiabatic fraction, the arrangement of adiabatic and conducting plates is crucial in determining the large-scale flow dynamics.

Key words: Bénard convection, mantle convection, sea ice

\section{Introduction}

Convection has a prominent place in our planet: the flows in the ocean, atmosphere and in the mantle are driven primarily by density differences. Convective flows are usually studied by using a simple model, i.e. Rayleigh-Bénard convection, the flow in a layer heated from below and cooled from above (Ahlers, Grossmann \& Lohse 2009; Lohse \& Xia 2010; Chillà \& Schumacher 2012; Xia 2013). For intermediate and high Prandtl number convection, which covers the geophysical flows mentioned above, heat is transported mainly by small-scale structures known as plumes. Even for very large temperature differences (Rayleigh numbers), and very turbulent flows, the

$\dagger$ Email address for correspondence: rostillamonico@g.harvard.edu 
plumes self-organize and a large-scale coherent flow arises. Plumes are ejected from preferential places in the boundaries, and impact at other points. This has immediate consequences for the flow and how it is felt. For example, in the context of the atmosphere, an upwelling region where plumes are ejected upwards could be thought of as a 'thermal', which birds and gliders seek to regain altitude. For the mantle, cold, downwelling regions on tectonic plates could represent places where the continents aggregate (Bott 1964).

While explorations of Rayleigh-Bénard convection have been extensive through the years, experiments and simulations have traditionally focused on thermally uniform boundary conditions. Both the mantle, in the shape of the differences between the oceanic and continental lithospheres, and the atmosphere and oceans, because of sea ice floating on water, have substantial inhomogeneity in their thermal boundary conditions. However, the effects of these inhomogeneities have barely been studied. In the mantle, it has been postulated that the difference in thermal conduction between continental and oceanic plates plays a crucial role in the topology of the mantle circulation, and this essentially determines the dynamics of the Wilson cycle. Sea ice comes in a wide range of topologies, and understanding how the fractures, leads and polynyas in ice affect the dynamics of oceanic and atmospheric circulation (Maykut 1982), and by extension global warming, is crucial in today's world where ice caps are disappearing. And while several studies for mixed temperature boundary conditions have been performed numerically either in two dimensions (Ripesi et al. 2014) or for lower Rayleigh numbers (Cooper, Moresi \& Lenardic 2013), no systematic analysis of the flow structure or heat transport in the turbulent regime was performed up to the work of Wang, Huang \& Xia (2017).

\section{Overview}

To help understand these issues, Wang et al. (2017) performed an experimental study of Rayleigh-Bénard flow with mixed thermal boundary conditions, insulating a fraction of the top plate with adiabatic patches. They used two experimental set-ups: a rectangular tank and a cylindrical tank, in an attempt to make their results as general as possible. These tanks were filled up with water. In both of these systems, several patterns of adiabatic and conducting boundary conditions were used, but in general their focus was on large adiabatic patches, with sizes comparable to the system size - each patch covering either a third of the cube's lid or a quarter of the cylinder lid. This in line with the simulations of Cooper et al. (2013), but contrasts with the approach by Ripesi et al. (2014), whose largest patches are a tenth of the plate size, and only get smaller.

Wang et al. (2017) first focus on the heat transfer, finding that for both geometries the leading-order effect happens through the fraction of the boundary which is adiabatic, and through the arrangement of the patterns. The adiabatic area affects the scaling behaviour of the heat transport, and for higher Rayleigh numbers the difference between a perfectly conducting plate and the mixed boundary conditions grows. However, the conducting efficiency is still higher than the fraction of conducting area - the authors find almost no decrease in heat transport for the $75 \%$ conducting cylinder when compared to the homogeneous case, and for the $25 \%$ conducting cylinder, the total heat transport is still over a half of the heat transport of the homogeneous case at the highest Rayleigh number. For the rectangular experiment, similar phenomena are seen. The conducting efficiency is higher than simply the fraction of the conducting area over the total area, and this efficiency tends to decrease with Rayleigh number. 

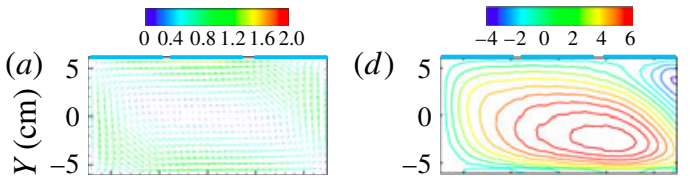

$(g)$
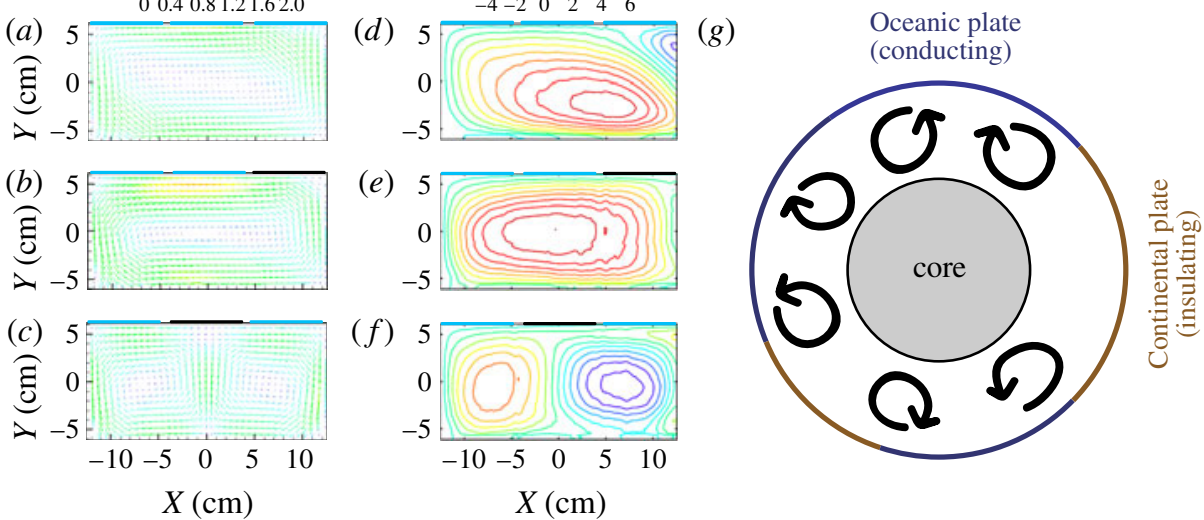

FIgURE 1. $(a-c)$ Time-averaged velocity fields for three configurations. The scale bar represents the modulus of the two in-plane components of velocity in units of $\mathrm{cm} \mathrm{s}^{-1}$. $(d-f)$ The corresponding contour maps of the streamfunction with the scale bar in units of $\mathrm{cm}^{2} \mathrm{~s}^{-1}$. The streamfunction values are set to zero at the cell boundaries. The contour interval is $0.6 \mathrm{~cm}^{2} \mathrm{~s}^{-1}$. Blue plates represent conducting boundaries and black plates represent adiabatic boundaries. The difference in the flow topology between the asymmetric, single roll, middle panel and the symmetric, two-roll bottom panel is apparent. Taken from Wang et al. (2017). ( $g$ ) Sketch outlining the basic mechanism for continental drift proposed by Bott (1964).

The authors also find that the probability density functions of the temperature fluctuations also seem to be insensitive to the insulating pattern, and depend more on the insulating area. However, the most significant effect of the adiabatic boundary conditions is seen in the large-scale wind. As shown in figure 1, in their rectangular cell, they can switch around between two flow topologies by rearranging the adiabatic patches with the same insulating fraction: either an asymmetric single roll when the patches are arranged asymmetrically or a symmetric double roll if they are arranged symmetrically. This confirms previous geophysical intuitions such as those of Bott (1964) on the role of quasi-adiabatic boundary conditions for convection.

The strength of the large-scale flow is also affected by both the adiabatic fraction and arrangement of the patterns. They find that the underlying wind is strongest when the patches are arranged antisymmetrically and the insulating area is larger. While this might be counter-intuitive, it is easy to rationalize by noting that the only way of removing heat is through the conducting boundaries - so there must be more circulation to remove all the heat, especially if the heat transport is higher than simply the fraction of conducting area multiplied by the homogeneous heat transport. The results confirm this, as the large enhancement in velocity is for the horizontal component and not so much for the vertical one.

\section{Future}

When compared to previous studies, the work by Wang et al. (2017) focuses on large adiabatic patches, as do the simulations of Cooper et al. (2013), while the simulations of Ripesi et al. (2014) in two dimensions, and their recent extension by Bakhuis et al. (2017) to three dimensions, have all focused on patches which have characteristic dimensions smaller than the distance between plates. The two latter numerical studies have shown that from a certain characteristic patch size, 
the effect on the flow dynamics becomes almost negligible in the turbulent regime. The three-dimensional work by Bakhuis et al. (2017) also showed that the effect of the shape of these patches is small, something which at first glance contradicts Cooper et al. (2013) and Wang et al. (2017). Therefore, a cross-over between two regimes must exist, one where large adiabatic patches which significantly affect the flow topology and another where small adiabatic patches whose effect is only present very close to the plates must exist. Understanding where that regime lies is crucial to understand geophysical flows, because while floating icebergs seem small in comparison with the ocean depth, tectonic plates could pretty much fall in between both categories, as the mantle is slightly less than $3000 \mathrm{~km}$ in extension, and if mantle convection is layered, the effective height is even smaller, while the characteristic length scale of plates is in the same range: thousands of kilometres. Another interesting avenue of study would involve a set-up with even larger insulating patches, in order to check whether these could totally prevent flow underneath certain continental plates, leading to cycles of buildup and sudden release of energy.

Finally, for geophysical applications, accounting for the effect of rotation or stabilization is crucial. Around the equator, Coriolis forces become dominant, and rotation drastically changes the flow topology (Greenspan 1990). Flow topology can also be changed by adding a second stabilizing field, as is the case in thermohaline convection (Schmitt 1994; Kellner \& Tilgner 2014; Yang, Verzicco \& Lohse 2016). Whether mixed thermal boundary conditions can produce the same significant effects as they do for the non-rotating non-stabilized Rayleigh-Bénard convection is an important question which merits attention.

\section{References}

Ahlers, G., Grossmann, S. \& Lohse, D. 2009 Heat transfer and large scale dynamics in turbulent Rayleigh-Bénard convection. Rev. Mod. Phys. 81, 503-537.

Bakhuis, D., Ostilla-Mónico, R., Van Der Poel, E. P., Verzicco, R. \& Lohse, D. 2017 Mixed insulating and conducting thermal boundary conditions in Rayleigh-Bénard flow. J. Fluid Mech. (submitted) arXiv:1702.06782.

Bотт, M. H. P. 1964 Convection in the Earth's mantle and the mechanism of continental drift. Nature 202, 583-584.

Chillà, F. \& Schumacher, J. 2012 New perspectives in turbulent Rayleigh-Bénard convection. Eur. Phys. J. E 35 (7), 1-25.

Cooper, C. M., Moresi, L.-N. \& Lenardic, A. 2013 Effects of continental configuration on mantle heat loss. Geophys. Res. Lett. 40 (11), 2647-2651.

Greenspan, H. P. 1990 The Theory of Rotating Flows. Breukelen Press.

Kellner, M. \& TIlgner, A. 2014 Transition to finger convection in double-diffusive convection. Phys. Fluids 26, 094103.

Lohse, D. \& XiA, K.-Q. 2010 Small-scale properties of turbulent Rayleigh-Bénard convection. Annu. Rev. Fluid Mech. 42, 335-364.

MAYKUT, G. A. 1982 Large-scale heat exchange and ice production in the central arctic. J. Geophys. Res. 87 (C10), 7971-7984.

Ripesi, P., Biferale, L., Sbragaglia, M. \& Wirth, A. 2014 Natural convection with mixed insulating and conducting boundary conditions: low- and high-Rayleigh-number regimes. J. Fluid Mech. 742, 636-663.

Schmitt, R. W. 1994 Double diffusion in oceanography. Annu. Rev. Fluid Mech. 26, 255-285.

WANG, F., HUANG, S.-D. \& XIA, K.-Q. 2017 Thermal convection with mixed thermal boundary conditions: effects of insulating lids at the top. J. Fluid Mech. 817, R1.

XIA, K.-Q. 2013 Current trends and future directions in turbulent thermal convection. Theor. Appl. Mech. Lett. 3, 052001.

YANG, Y., Verzicco, R. \& LohSE, D. 2016 From convection rolls to finger convection in doublediffusive turbulence. Proc. Natl Acad. Sci. USA 113 (1), 69-73. 